\title{
Comparative study of antibacterial activity of vancomycin and chemically treated chitosan prepared from shrimp (Macrobrachium rosenbergii) waste
}

\author{
Rashna Gulshan 1, Md. Tanvir Sarwar ${ }^{1}$, Md. Abdullah Al Mamun², Md. Jahangir Alam², \\ Sabir Hossain ${ }^{3}$, M. Masihul Alam ${ }^{1,}$ \\ ${ }^{1}$ Dept. of Applied Nutrition and Food Technology, Islamic University, Kushtia-7003, Bangladesh \\ ${ }^{2}$ Dept of Genetic Engineering and Biotechnology, Shahjalal University of Science \& Technology, Sylhet-3114, Bangladesh \\ ${ }^{3}$ Biochemistry and Molecular Biology Division, Jahangirnagar University, Savar, Dhaka, Bangladesh
}

\section{Email address:}

alam.anft@gmail.com (M. M. Alam)

\section{To cite this article:}

Rashna Gulshan, Md. Tanvir Sarwar, Md. Abdullah Al Mamun, Md. Jahangir Alam, Sabir Hossain, M. Masihul Alam. Comparative Study of Antibacterial Activity of Vancomycin and Chemically Treated Chitosan Prepared from Shrimp (Macrobrachium Rosenbergii) Waste. International Journal of Nutrition and Food Sciences. Vol. 2, No. 6, 2013, pp. 307-311. doi: 10.11648/j.ijnfs.20130206.17

\begin{abstract}
Chitosan is derived from exoskeleton of crustaceans (such as crabs and shrimp) and cell walls of fungi has a number of commercial and possible biomedical uses is produced commercially by deacetylation of chitin is a linear polysaccharide composed of randomly distributed $\beta$-(1-4)-linked D-glucosamine (deacetylated unit) and N-acetyl-Dglucosamine (acetylated unit). The structural element This study was conducted to compare the antibacterial activity of chitosan with the combination of chitosan and vancomycin against gram negative Escherichia coli and gram positive Staphylococcus aureus. Optical density (OD) value measurement technique was undertaken to measure antibacterial activity. After incubation, turbidimetric measurements of bacterial growth of sets of test tubes were taken after every 4 hours, for 48 hours and then compared. For $S$. aureus and chitosan the average absorbance were found as $0.448,0.457$, $0.492,0.532,0.582,0.645,0.683,0.724,0.751,0.793,0.840$, and 0.896 , while for S. aureus with both chitosan and vancomycin, absorbance were $0.267,0.286,0.321,0.346,0.386,0.431,0.467,0.475,0.509,0.541,0.565$, and 0.629 . Again for E. coli and chitosan the average absorbance were found as $0.407,0.429,0.464,0.506,0.564,0.6,0.645,0.703,0.756$, $0.815,0.850$, and 0.901 , while E. coli with both chitosan and vancomycin, absorbance were $0.213,0.233,0.322,0.347$, $0.409,0.446,0.475,0.511,0.545,0.582,0.628$, and 0.647 . Both of these revealed that chitosan and vancomycin together possess higher antibacterial activity against gram positive and gram-negative bacteria than chitosan solitarily used. Statistical analysis of the both study also represented in Mean \pm 3 SD. Comparison of the antibacterial activity against $E$. coli and S. aureus revealed that chitosan and vancomycin posses similar antibacterial activity against gram positive and gram negative bacteria.
\end{abstract}

Keywords: Antibacterial activity, Chitosan, Vancomycin

\section{Introduction}

Chitosan is a linear polysaccharide that consists of $(1,4)$ linked 2-amino-deoxy-N- D-glycan. It is a deacetylated form of chitin, the second most abundant polysaccharide found in nature after cellulose [1]. It also exists naturally in a few species of fungi; insects and yeast [2]. Extensive studies have been conducted on the activity of vancomycin on gram positive and gram negative bacteria. To facilitate the genetic investigation, entire genome sequences of the archetypal VRSA (Mu50) and vancomycin-susceptible MRSA strains N315, EMRSA 16 and COL were compared
[3]. Their research helped to understand the mechanism(s) of vancomycin resistance in S. aureus Mu50 and other VRSA strains. The antimicrobial activity of chitosan with that of combined chitosan and vancomycin was compared which ultimate goal was to increase effectiveness of narrow spectrum antibiotics applied to preserve food items, may apply as antimicrobial agent in pharmaceuticals and may also find uses in agriculture as a more active seed coating, fertilizer, controlled agrochemical release agent. In drug industry it may be used to formulate weight loosing supplement. Chitosan production from shrimp waste certainly save the environment from serious pollution [4] may be used as water purification agent in municipal area 
where people always face serious water problem. After considering the applicability of chitosan and its possible development by vancomycin as well as narrow spectrum antibiotic, this research work has been undertaken.

Chitin and chitosan in vitro show antibacterial and antiyeast activities. One of chitosan derivatives, i.e., Ncarboxybutyl chitosan, was tested against 298 cultures of different pathogenic microorganisms that showed bacteriostatic and bactericidal activities, and there were marked morphological alterations in treated microorganisms when examined by electron microscopy [5]. Conversely, growth inhibition and inactivation of mould and yeasts seem to depend on chitosan concentration, $\mathrm{pH}$, and temperature [6].

The antimicrobial activity of chitosan varies depending on their physical properties (degree of deacetylation (DD), and molecular weight), solvent, microorganism species and source. The antimicrobial activity is reported to vary depending on the methods involved in preparation of different DD and molecular weight of chitosan $[7,8]$. The antimicrobial action of chitosan is influenced by intrinsic and extrinsic factors such as the type of chitosan (e.g., plain or derivative), degree of chitosan polymerization, host nutrient constituency, substrate chemical and/or nutrient composition, and environmental conditions such as substrate water activity [9]. In an extensive research on the antimicrobial activity of chitosan prepared from shrimp against E. coli, it was found that higher temperature and acidic $\mathrm{pH}$ of foods increased the bactericidal effect of chitosan [10]. The mechanism of chitosan antibacterial action involving a cross-linkage between polycations of chitosan and the anions on the bacterial surface that changes membrane permeability and has been approved as a food additive in Japan and Korea since 1983 and 1995 respectively [11].

Based on the above investigation the present study was undertaken to compare whether chitosan is more active than vancomycin and vice-versa against E. coli and Staphylococci. The study of the anti-bacterial activity of chemically deacylated chitosan prepared from shrimp and the comparison of anti bacterial activity of chitosan with the combination of chitosan and a narrow spectrum antibiotic vancomycin

\section{Materials and Methods}

Chitin is extracted from crustacean shell waste such as crab, shrimp, lobster, and crawfish. We prepared chitin from shrimp shell (Macrobrachium rosenbergii) locally known as Galda chinri.

\subsection{Chitosan Isolation}

Isolation of chitosan from shirmp shell wastes involves four traditional steps demineralization (DM), deproteinization (DP), decolorization (DC), and deacetylation (DA). The method was modified from No and Meyers, (1995) [12].
Escherichia coli and Staphylococcus aureus strains were tested for the antimicrobial activity of chitosan and vancomycin. Pure culture of Staphylococcus aureus was previously collected from ICDDRB, Dhaka and cultured in Mac Conkey and nutrient agar media respectively and maintained in the lab.

\subsection{Experimental Procedure}

The study was carried out by broth dilution method and used turbidity estimations to determine the growth characteristics of a bacterial culture with or without chitin or Three percent of concentrated (99\%) acetic acid was prepared in a conical flask with distill water to dissolve the powder. $7.5 \mathrm{gm}$ chitosan was taken into two sterile test tubes and $50 \mathrm{ml}$ of $3 \%$ acetic acid was poured into each of these gradually. To increase solubility of the solution was stirred; heat was applied in water bath at $400 \mathrm{C}$. The solution was left at room temperature for 3 days. Stirring and heat was applied in this time with a spirit lamp. Nutrient broth (N.B) was used as culture media in test tubes.

Nine ml of nutrient broth media was taken in each of fourteen 14-test tubes. Each test tube was always capped with screw to avoid contamination. Three test tubes were used as standard in spectrophotometer measurement for blank calculation contains media. The upper soluble portion of chitosan sample was added to NB medium in test tube, the pellet portion of the test tube was not taken. $0.5 \mathrm{ml}$ supernatant chitosan solution was taken by micropipette and dispended in each test tube. The $\mathrm{pH}$ of chitosan with medium was also adjusted between 6.2-6.5 range to ensure optimum growth of microorganism using $\mathrm{NaOH}$ solution. Five vancomycin discs were added in each test tube.

Two test tubes containing $9 \mathrm{ml} \mathrm{N} . \mathrm{B}$ media and $0.5 \mathrm{ml}$ chitosan solution was mixed. One loop full staphylococci. The loop head was immersed into the broth and mixed well. The same process was followed for other two test tubes with gram negative bacteria. Another two test tubes containing N.B media, chitosan and vancomycin were taken and inoculated them with gram positive bacteria and another two tubes with gram negative bacteria in the same way. Absorbance of Nutrient broth with S. aureus, and E. Coli was measured separately at $640 \mathrm{~nm}$. Chitosan was included separately with the both strain separately for the measurement of activity. Vancomycin was incorporated in broth chitosan and the two organisms in which equal concentration of inoculum was used. In each case blank was prepared. After successful inoculation the test tubes were incubated in an incubator at $37^{\circ} \mathrm{C}$. After each 4 hours interval measurements were taken by spectrophotometer up to $48 \mathrm{hrs}$. Absorbance or OD value can also refer to index of refraction [13]. This technique is based on the principle that small particles, such as bacteria, scatter a beam of light passed through the cell suspension. The amount of light scattering is proportional to the concentration of particles in the suspension; because a pure culture of single cells is relatively uniform in size (mass). The number of cells can 
be measured from the amount of light that reaches a photoelectric cell after passing through the cell suspension. As the bacterial cell population increases, the amount of transmitted light decreases, increasing the absorbance reading on the spectrophotometer. These sequences were repeated for other samples and the respective blank was changed in blank and the absorbance was recorded. O.D values were taken after every 4 hours for 48 hours.

\section{Results and Discussions}

The recorded absorbance taken for Staphylococcus aureus and E. coli are given in the table 1.

Table1. OD values for $S$. aureus (4h-48h)

\begin{tabular}{llllllllllllll}
\hline \multicolumn{1}{c}{ Description } & \multicolumn{1}{c}{ Average OD values } \\
\hline Hours of incubation & 4 & 8 & 12 & 16 & 20 & 24 & 28 & 32 & 36 & 40 & 44 & 48 \\
Chitosan + S. aureus & 0.448 & 0.457 & 0.492 & 0.532 & 0.582 & 0.645 & 0.683 & 0.724 & 0.751 & 0.793 & 0.840 & 0.896 \\
Chitosan + S. aureus + Antibiotic & 0.267 & 0.286 & 0.321 & 0.346 & 0.386 & 0.431 & 0.467 & 0.475 & 0.509 & 0.541 & 0.565 & 0.629 \\
\hline
\end{tabular}

The OD value measured for Staphylococcus aureus with chitosan demonstrated far higher absorbance than chitosan and vancomycin together. So it is clearly evident that chitosan and vancomycin together possess high level antibacterial property against $S$. aureus, while chitosan alone possess lower level of activity. Graphical presentation of the measured OD values for $S$. aureus has been shown in figure 1. Where V1 represents absorbance in Chitosan and staphylococcus and V2 represents the same included antibiotic. The error bars shows symmetrical distribution at Mean \pm 3SD level.

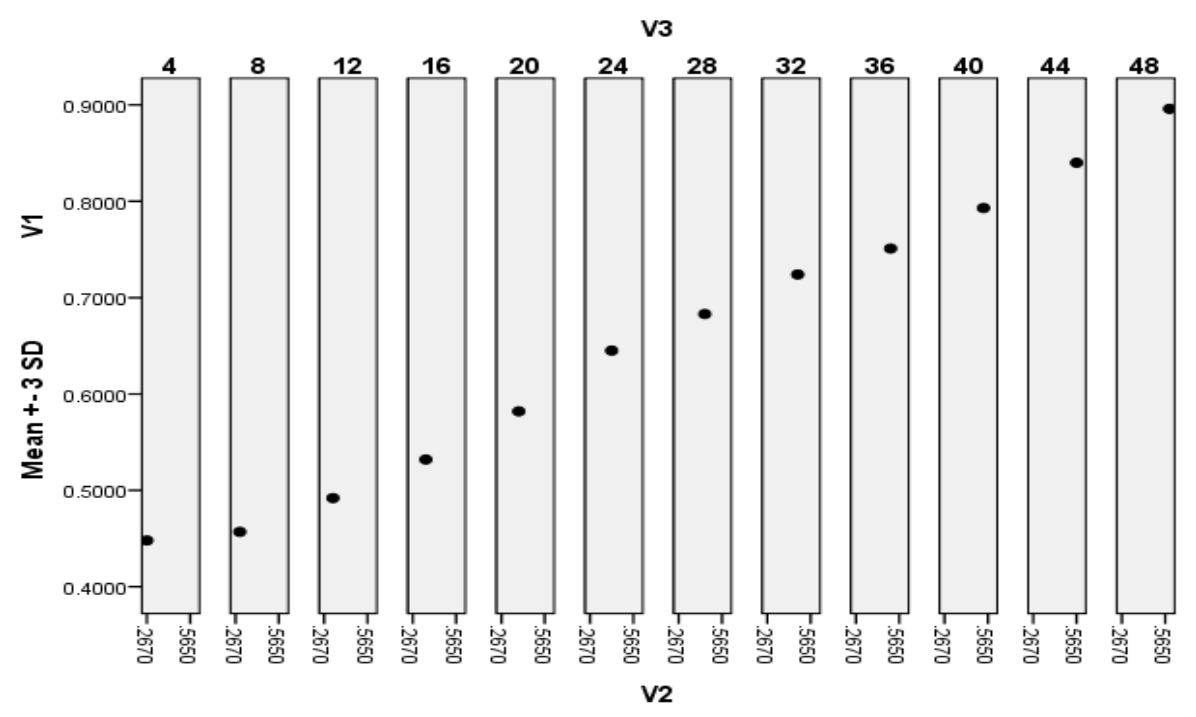

Figure 1. Growth curve for S .aureus + chitosan and S. aureus + chitosan + vancomycin

Table 2. OD values for E. coli (4h-48h)

\begin{tabular}{|c|c|c|c|c|c|c|c|c|c|c|c|c|}
\hline Description & & & & & & Average & D value & & & & & \\
\hline $\begin{array}{l}\text { Hours of } \\
\text { incubation }\end{array}$ & 4 & 8 & 12 & 16 & 20 & 24 & 28 & 32 & 36 & 40 & 44 & 48 \\
\hline Chitosan + E. coli & 0.407 & 0.429 & 0.464 & 0.506 & 0.564 & 0.600 & 0.654 & 0.703 & 0.756 & 0.815 & 0.85 & 0.901 \\
\hline $\begin{array}{l}\text { Chitosan + E. coli } \\
\quad+\text { Antibiotic }\end{array}$ & 0.213 & 0.233 & 0.322 & 0.374 & 0.409 & 0.446 & 0.475 & 0.511 & 0.545 & 0.582 & 0.628 & 0.647 \\
\hline
\end{tabular}

The OD value measured for Escherichia coli with chitosan demonstrated far higher absorbance than chitosan and vancomycin together.

It is clearly evident that chitosan and vancomycin together possess high level antibacterial property against E. coli, while chitosan solitarily possess lower level of activity. Graphical presentation of the measured O.D. values for $E$. coli has been shown in figure 2. Where V1 represents 
absorbance in Chitosan and E. coli and V2 represents the same included antibiotic. The graph shows symmetrical distribution at Mean \pm 3 SD level.

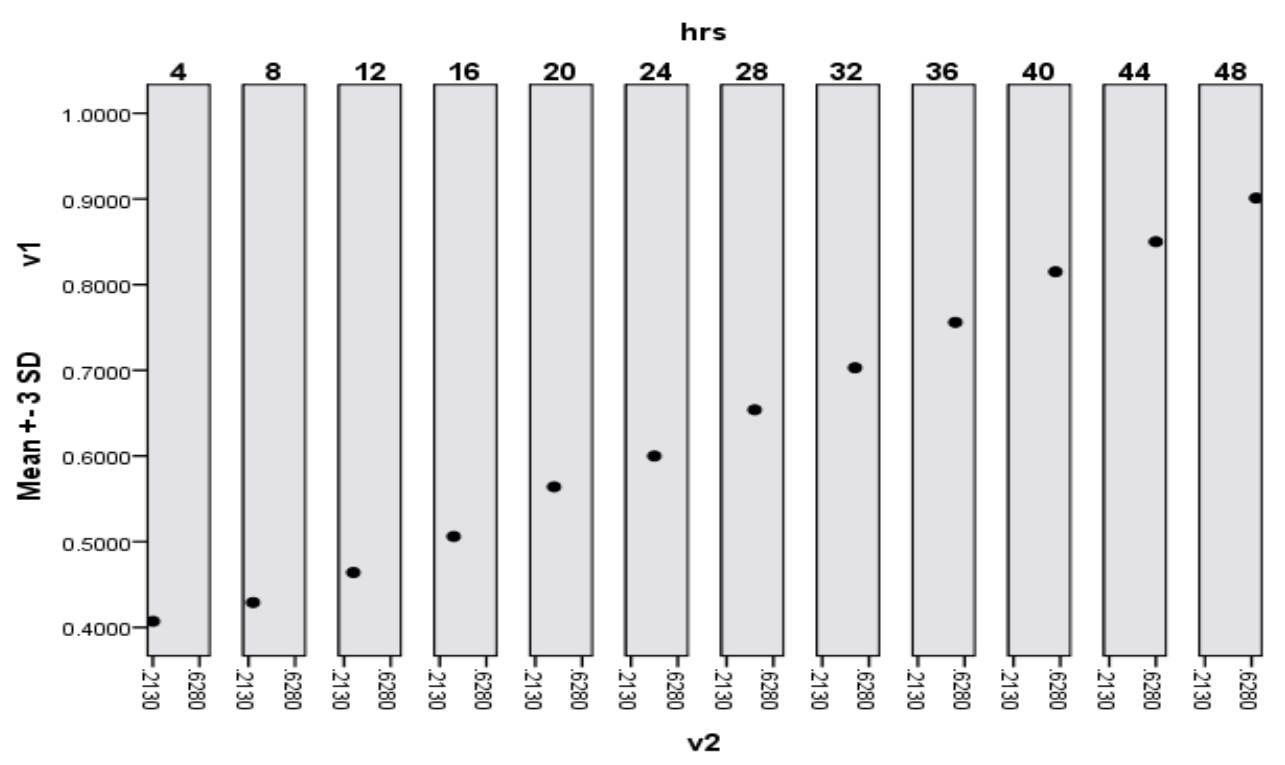

Figure 2. Shows growth curve for E. coli + chitosan and E. coli + chitosan + vancomycin

The growth of E. coli and staphylococcus with chitosan and vancomycin lies far behind than the growth curve of $\mathrm{E}$. coli and staphylococcus with chitosan reveals growth of E.coli and staphylococcus is retarded more by the addition of vancomycin with chitosan. Comparative study of antibacterial activity of chitosan and vancomycin against $\mathrm{E}$. coli and S. aureus has shown in figure. 3.

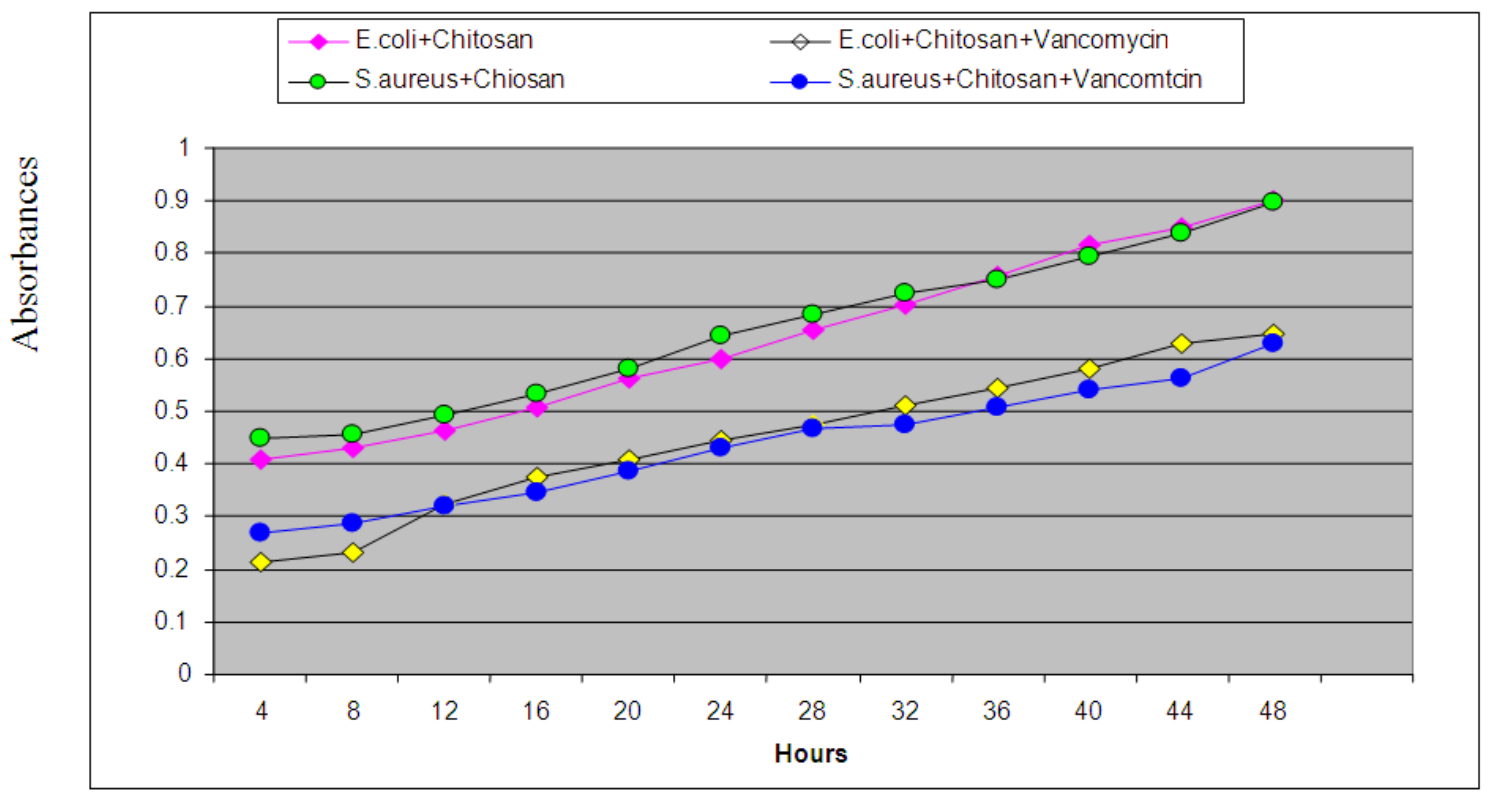

Figure 3. Comparison of the growth curves for E. coli and S. aureus

The figure 3 reveals that in presence of vancomycin at first the growth of gram negative E. coli was lower than that of gram positive $S$. aureus. After 12 hours growth of both the bacteria was same. E. coli showed slightly higher growth than S. aureus. Although in some points OD value for E. coli and S. aureus were approximately same. The efficacy of many antibiotics for treatment of infections has become quite limited due to the development of resistance and the threat from antimicrobial-resistant organisms is accumulating and accelerating [14]. Also, the development of resistance to mono therapy is a common problem and dual antimicrobial coverage is often a necessity in 
Pseudomonas infections [15]. Attempts have been made to deal with this problem by using combination therapy with success [16]. Present study will meet the necessary requirement to understand mechanism of activity of natural polysaccharide which will solve best use of antibacterial agents.

\section{Conclusion}

The activity of chitosan is higher against gram positive bacteria than gram negative bacteria. In this study chitosan and vancomycin together showed slightly raised antibacterial effect against gram negative $E$. coli, the difference between antibacterial activity against both the gram positive $S$. aureus and gram negative $E$. coli very little as negligible. So from this research it have been found that, chitosan and vancomycin possess approximately equal antibacterial activity against gram positive and gram negative bacteria but when they used together as antimicrobial agent they work more effectively than chitosan or vancomycin alone. In table-1 it has been shown that the average OD value of chitosan and aureus is higher than chitosan and aureus and vancomycin together. It is also shown that the existence of a synergistic effect between chitosan and vancomycin antibiotic. Since chitosan itself acts as an antibiotic, it can certainly increase the activity of vancomycin, which would take the antibiotic technology forward one step. Chitosan and vancomycin together can also create advanced ways to produce more effective preservatives.

\section{Acknowledgment}

The research was jointly supported and organized by Department of Applied Nutrition and Food Technology Islamic University, Bangladesh and Dept. of Genetic Engineering and Biotechnology, Shahjalal University of Science \& Technology, Sylhet, Bangladesh.

\section{References}

[1] Jayakumar, R.; New, N.T.; Tokura, S.; Tamura, H. Sulfated Chitin and Chitosan as novel biomaterial. Int. J. Biol. Macromol., 40, 175-181, 2007.

[2] Austin P.R., Brine C.J., Castle J.E. ans Zikakis J.P. Chitin: New facets of research. Science, 749, 1981.

[3] Matthew B. Avisona, Peter M. Bennettb, Robin A. Howec and Timothy R. Walsh. Preliminary analysis of the genetic basis for vancomycin resistance in Staphylococcus aureusstrain Mu50. J. Antimicrob. Chemother. 49 (2), 255$260,2002$.
[4] Abu Tareq, M. Masihul Alam, Md. Salim Raza, Md. Tanvir Sarwar, Z. Fardous, Chowdhury, M. Sabir Hossain. Comparative study of antibacterial activity of chitin and chemically treated chitosan prepared from shrimp (Macrobrachium rosenbergii) shell waste. Int. Res. J. of Microbiol. (IRJM) 2013 (2013)

[5] Koide S.S. Chitin-Chitosan: properties, Benefits and Risks. Nutrition Research. 18(6), 1091-1101, 1998.

[6] Rout S. K. Physicochemical, Functional, and Spectroscopic analysis of crawfish chitin and chitosan as affected by process modification. Dissertation, 2001.

[7] Qin CQ, Li HR, Xiao Q, Liu Y, Zhu JC, Du YM. Watersolubility of chitosan and its antimicrobial activity. Carbohydrate Polymers. 63, 367-374, 2006.

[8] Chen Y M, Chung YC, Wang LW, Chen KT, Li SY. Antibacterial activity of chitosan- based matrixes on oral pathogens. Health A Tox Hazard Subst Environ Eng. $J$. Environ Sci. 37, 1379-1390, 2002.

[9] Cuero, R.G. Antimicrobial action of exogenous chitosan. EXS. 1999. 87.p.315-333. Del Blanco L.F., Rodriguez M.S., Schulz P.C., Agullo E. Influence of the deacetylation degree on chitosan emulsification properties. Colloid Polymer Science. 277, 1087-1092. 1999.

[10] Abu Tareq, M. Masihul Alam, Md. Salim Raza, Md. Tanvir Sarwar, Z. Fardous, Alamgir Z. Chowdhury, Sabir Hossain. Comparative study of antibacterial activity of chitin and chemically treated chitosan prepared from shrimp (Macrobrachium rosenbergii) shell waste International Journal of Virology Microbiology (JVM)Vol. 2013. 1-9, 2013.

[11] Weiner M. L. An overview of the regulatory status and of the safety of chitin and chitosan as food and pharmaceutical ingredients. In: Brine, C.J., Sandford, P.A., Zikakis, J.P. (Eds.).1992.

[12] No, H. K. \& Meyers, S. P. "Preparation and Characterization of Chitin and Chitosan-A Review," Journal of Aquatic Food Product Technology. 4(2). 27-52. 1995

[13] Zizewitz, Paul W., New York, N. Y. Glencoe/ M.CGrawHill,Glencoe Physics. ISBN 0028254732. P. 395. 1999.

[14] Bosso JA. The antimicrobial armamentarium: evaluating current and future treatment options. Pharmacotherapy. 25,55S-62S, 2005.

[15] Ling TKW, Xiong J, Yu Y. et al. Multicenter antimicrobial susceptibility survey of Gram-negative bacteria isolated from patients with community-acquired infections in the People's Republic of China. Antimicrob Agents Chemother. 50, 374-378, 2006.

[16] George ME, Robert C, Moellering JR. Antimicrobial Combinations. Antibiotics in Laboratory Medicine 4th ed. Baltimore: The Williams \& Wilkins Co. 1996. 